\title{
EFFECT OF EXOGENOUS APPLICATION OF MICRONUTRIENTS ON GROWTH AND PRODUCTIVITY OF COTTON (Gossypium hirsutum L.) CROP
}

\author{
EFEITO DA APLICAÇÃO EXÓGENA DE MICRONUTRIENTES NO CRESCIMENTO E \\ PRODUTIVIDADE DA CULTURA DE ALGODÃO (Gossypium hirsutum L.)
}

\author{
Fahad AJMAL ${ }^{1}$; Muhammad AKRAM ${ }^{2}$; Rana Muhammad IQBAL ${ }^{3}$; Mubshar HUSSAIN ${ }^{4}$; \\ Muhammad Waqar HASSAN ${ }^{3}$; Muhammad ABDULLAH ${ }^{1}$; Allah WASAYA ${ }^{5}$ \\ 1. Cholistan Institute of Desert Studies, The Islamia University of Bahawalpur, Pakistan; 2. Department of Environmental Sciences, \\ COMSATS Institute of Information Technology, Vehari Pakistan; 3. University College of Agriculture and Environmental Sciences, The \\ Islamia University of Bahawalpur, Pakistan; 4. Department of Agronomy, Bahauddin Zakariya University, Multan Pakistan, 5. College of \\ Agriculture, Sub Campus Bahadur Layyah, Bahauddin Zakariya University Multan Pakistan.* akramcp@ gmail.com
}

\begin{abstract}
Micronutrients play a vital role in the growth and productivity of cotton crop. A study was carried out to access the exogenous application of micronutrients on growth and yield of cotton crop. The experiment was comprised of nine treatments as $\mathrm{T}_{0}$ (control), $\mathrm{T}_{1}\left(\mathrm{Fe}\right.$ chelated), $\mathrm{T}_{2}(\mathrm{~B}), \mathrm{T}_{3}(\mathrm{Mo}), \mathrm{T}_{4}\left(\mathrm{CuSo}_{4}+\mathrm{ZnSo}_{4}+\mathrm{MnSo}_{4}\right), \mathrm{T}_{5}\left(\mathrm{CuSo}_{4}+\mathrm{ZnSo}_{4}+\mathrm{MnSo}_{4}+\right.$ Fe chelated $), \mathrm{T}_{6}\left(\mathrm{CuSo}_{4}+\mathrm{ZnSo}_{4}+\mathrm{MnSo}_{4}+\mathrm{B}\right), \mathrm{T}_{7}\left(\mathrm{CuSo}_{4}+\mathrm{ZnSo}_{4}+\mathrm{MnSo}_{4}+\mathrm{Mo}\right)$ and $\mathrm{T}_{8}\left(\mathrm{CuSo}_{4}+\mathrm{ZnSo}_{4}+\mathrm{MnSo}_{4}+\mathrm{Fe}\right.$ chelated + B). Data on different growth attributes showed that there was significant positive increase with the application of micronutrients. Leaf area was increased after applying micronutrients at 99 days after sowing (DAS) and then a decreasing trend was observed. Chlorophyll contents were increased at 81 DAS and then decreased towards the final harvest. Similarly, different yield components showed that seed cotton yield were significantly increased with the application of Fe, B, Mo, Zn, $\mathrm{Cu}$ and $\mathrm{Mn}$ compared to control treatment. Earliness index, mean maturity date and production rate index were increased significantly after combined use of foliar spray of $\mathrm{Zn}, \mathrm{Cu}, \mathrm{Mn}$ and $\mathrm{Mo}$.
\end{abstract}

KEYWORDS: Growth. Yield. Micronutrients. Cotton.

\section{INTRODUCTION}

Micronutrients are very essential and their soil application of $\mathrm{Zn}, \mathrm{B}, \mathrm{Fe}, \mathrm{Mn}$, and $\mathrm{Cu}$ particularly on calcareous soils is less efficient, as these nutrients remain unavailable to plant roots due to the higher $\mathrm{pH}$ of these soils (SAJID et al., 2008). Another approach under such conditions is foliar application of nutrients (RAB; HAQ, 2012) primarily for two reasons. First, it reduces the effects of soil $\mathrm{pH}$ on the availability of these nutrients (ALI, 2012) and secondly, it is more efficient and less costly (ALI et al., 2007). The foliar application of nutrients has gained significant interest for agricultural scientist throughout the world as it is successful and cost-effective technology (ALI, 2012; LIEW et al., 2012).

Micronutrients are essential for normal growth and development of plants as zinc is necessary for plants in some of their enzymatic systems. Manganese play role in oxidation reduction reactions in plants especially with iron and nitrogen metabolism as the iron is essential for chlorophyll formation (ABRO et al., 2004). Micronutrients are vital for improvement of both qualitative and quantitative yield in oil seed crop (BAHRANI; POURREZA, 2014).
Foliar application of growth regulators and micronutrients are effective when they were sprayed alone, as boron application increased the seed cotton yield (DONG, 1995; HALLIKERI et al., 2002). Boron is an essential micronutrient for higher plants whose deficiency affects the meristematic development, resulting in stunted growth (MARSCHNER, 1995). Application of zinc to cotton crop promotes boll retention and consequently increases the seed cotton yield (MANGAL; PRASAD, 1998).

Cotton (Gossypium hirsutum L.), also known as queen of fibre, is the leading fibre crop of the world and is grown over an area of 3106 thousand hectares, with a production of 12968 thousand bales and average yield of $695 \mathrm{~kg}$ per hectare in the year of 2009 to 2010. Cotton is the main cash crop of Pakistan and is grown primarily both for fiber and oilseed. According to annual report of ECONOMIC SURVEY OF PAKISTAN (2009-2010) the cotton production was estimated 12.7 million bales for 2009 to 2010 , higher by $7.4 \%$ over the last year's production of 11.8 million bales. During these years, cotton contributes $8.6 \%$ of the value added in agriculture and approximately $1.8 \%$ in GDP. 
Keeping in view the role of nutrients in improving the yield of crops, the foliar application of micronutrients has been emphasized these days. Since little information is available on application of micronutrients through foliar sprays and consequently their effect on morphology and yield of cotton, hence, the present study was carried out to evaluate the effect of micronutrients as growth regulator and to determine their effect on growth and yield of cotton crop.

\section{MATERIAL AND METHODS}

An experiment was conducted to study the foliar application of micronutrients on the growth and yield of cotton at Cholistan Institute of Desert Studies, Baghdad-ul-Jadeed Campus, The Islamia University of Bahawalpur, Pakistan, during the year 2010-11. The experiment was laid out in randomized complete block design and there were three replications of each treatment.

The experiment was comprised of nine treatments as $\mathrm{T}_{0}$ (control), $\mathrm{T}_{1}$ (Fe chelated), $\mathrm{T}_{2}(\mathrm{~B}), \mathrm{T}_{3}$ (Mo), $\mathrm{T}_{4}\left(\mathrm{CuSo}_{4}+\mathrm{ZnSo}_{4}+\mathrm{MnSo}_{4}\right), \mathrm{T}_{5}\left(\mathrm{CuSo}_{4}+\right.$ $\mathrm{ZnSo}_{4}+\mathrm{MnSo}_{4}+\mathrm{Fe}$ chelated $), \mathrm{T}_{6}\left(\mathrm{CuSo}_{4}+\mathrm{ZnSo}_{4}+\right.$ $\left.\mathrm{MnSo}_{4}+\mathrm{B}\right), \mathrm{T}_{7}\left(\mathrm{CuSo}_{4}+\mathrm{ZnSo}_{4}+\mathrm{MnSo}_{4}+\mathrm{Mo}\right), \mathrm{T}_{8}$ $\left(\mathrm{CuSo}_{4}+\mathrm{ZnSo}_{4}+\mathrm{MnSo}_{4}+\right.$ Fe chelated $\left.)+\mathrm{B}\right)$. Copper sulphate, zinc sulphste and manganese sulphate were applied as a source of $\mathrm{Cu}, \mathrm{Zn}$ and $\mathrm{Mn}$. One percent solution of each micronutrient was applied regularly at 20 days intervals. The seed rate used was $25 \mathrm{~kg}$ per hectare and fertilizers $\left(75 \mathrm{~kg} \mathrm{~N}, 30 \mathrm{~kg} \mathrm{P}_{2} \mathrm{O}_{5}\right.$ and $50 \mathrm{~kg}$ $\mathrm{K}_{2} \mathrm{O} / \mathrm{ha}$ ) was applied at regular intervals of 20 days. Data on growth and yield parameters were recorded from 5 randomly selected plants in each treatment plot measuring $36.45 \mathrm{~m}^{2}$ (having 78 plants in 6 rows with $67.5 \times 75 \mathrm{~cm}$ geometry). Seed cotton yield $(\mathrm{kg} / \mathrm{ha})$ was calculated from whole plot. All other recommended production and protection practices were uniformly applied. Weeding was performed after regular intervals of 10 days and irrigation was performed by canal water at 15 days interval.

The data on different growth components as plant height was measured with the help of scale from the ground surface to the plant terminal. Number of leaves was taken by counting the total number of leaves of four tagged plants in each plot, and then average of these four tagged plants was calculated. Number of nodes present on the main stem of the four tagged plants was counted, and average was worked out. Leaf area was determined by leaf area meter and chlorophyll contents in plant leaf were estimated by chlorophyll meter (SPAD-502 value). Number of bolls of tagged plant was also counted regularly and then average was taken. Earliness index was calculated with the help of following formula as proposed by Singh (2003).

Earliness Index $(\%)=\frac{\text { Weight of seed cotton from first pick }}{\text { Total seed cotton weight from all picks }}$

Mean maturity date in cotton was calculated by the formula as proposed by Christidis and Harrison (1955).

Mean Maturity Date $(M M D)=\frac{\left(\mathrm{W}_{1} \times \mathrm{H}_{1}\right)+\left(\mathrm{W}_{2} \times \mathrm{H}_{2}\right)+\ldots \ldots \ldots+(\mathrm{Wn} \times \mathrm{Hn})}{\mathrm{W}_{1}+\mathrm{W}_{2}+\ldots \ldots \ldots+\mathrm{Wn}}$

Where $\mathrm{W}=$ weight of seed cotton, $\mathrm{H}=$ number of days from planting to harvest, $1,2 \ldots n=$ consecutive periodic harvest number.

Saleem et al. (2010) calculated that production rate index was calculated from total seed cotton weight divided by the mean maturity date.

Production Rate Index (g/day) $=\frac{\text { Total seed cotton weight }(\mathrm{g})}{\text { Mean maturity date (day) }}$

Statistical analysis

Data collected during the course of study were statistically analyzed using

MSTAT-C programme (ANONYMOUS, 1986) for analysis of variance and significant means were separated using least significant difference test (LSD) at $5 \%$ probability level (STEEL et al., 1997). Regression and correlation analysis was performed using MS Excel program against different variables.

\section{RESULTS}

Highest plant height $(86.25 \mathrm{~cm})$ was recorded at 117 days after sowing (DAS) in $\mathrm{T}_{8}$ treatment $(\mathrm{Fe}+$ $\mathrm{B}+\mathrm{Mo}+\mathrm{Zn}+\mathrm{Cu}+\mathrm{Mn})$, which was closely followed by $\mathrm{T}_{7}, \mathrm{~T}_{6}$ and lowest $(63.83 \mathrm{~cm})$ was recorded in control, where no micronutrient was applied (Table 1). Number of leaves in all the treatments continued to increase from 45 DAS up to 108 DAS and then gradually declined towards the final harvest. At 108 days of sowing, highest number of leaves (60.33) was recorded in $\mathrm{T}_{6}$ treatment, while lowest number of leaves (42.83) was recorded in control (Table 2). 
Effect of exogenous application...

At 72 DAS, treatment $T_{5}$ attained the highest number of nodes (14) and in control lowest number of nodes (11.25) was recorded. After 108 DAS, highest number of nodes per plant (15.69) was produced under the application of $\mathrm{T}_{8}$ treatment and control had the lowest number of nodes i.e. 13.65 (Table 3). The treatment $\mathrm{T}_{8}(\mathrm{Fe}+\mathrm{B}+\mathrm{Mo}+\mathrm{Zn}+\mathrm{Cu}+\mathrm{Mn})$ contributed to the maximum leaf area i.e. $55.55 \mathrm{~cm}^{2}$, and in control (no treatment) showed the minimum leaf area as $44.25 \mathrm{~cm}^{2}$, at 99 DAS (Table 4). Highest leaf area $\left(50.8 \mathrm{~cm}^{2}\right)$ was attained by treatment $\mathrm{T}_{8}$ while least $\left(39.13 \mathrm{~cm}^{2}\right)$ was recorded in control where no micronutrient was applied at 108 DAS (Table 4).

The Chlorophyll content in all the treatments continued to increase from 45 to 81 DAS, and then gradually declined towards the final harvest. There was significant variation $(\mathrm{p}<0.05)$ in chlorophyll contents (SPAD-value) from 54 DAS to onward and at 81 DAS it ranged from 49.35 to 56.77 in control and $\mathrm{T}_{8}$ treatment $(\mathrm{Fe}+\mathrm{B}+\mathrm{Mo}+\mathrm{Zn}+\mathrm{Cu}+\mathrm{Mn})$ respectively (Table 5). Data regarding number of bolls per plant, indicate that maximum number of bolls (9.83) was recorded in $\mathrm{T}_{8}$ treatment $(\mathrm{Fe}+\mathrm{B}+\mathrm{Mo}+$ $\mathrm{Zn}+\mathrm{Cu}+\mathrm{Mn}$ ), which was closely followed by $\mathrm{T}_{6}$ $(\mathrm{Cu}+\mathrm{Zn}+\mathrm{Mn}+\mathrm{B})$. The minimum number of bolls per plant (5.17) was recorded in control where no micronutrient was applied. Treatment $\mathrm{T}_{8}(\mathrm{Fe}+\mathrm{B}+$ $\mathrm{Mo}+\mathrm{Zn}+\mathrm{Cu}+\mathrm{Mn}$ ) yielded the highest number of bolls per plant (9.83) and control treatment had the lowest value (Table 6).

Number of cotton seed per boll increase significantly and in treatment $\mathrm{T}_{8}$ there was $34.63 \%$ increase was recorded comparative to control. Seed cotton yield was highest $(2900 \mathrm{~kg} / \mathrm{ha})$ in $\mathrm{T}_{8}$ treatment $(\mathrm{Fe}+\mathrm{B}+\mathrm{Mo}+\mathrm{Zn}+\mathrm{Cu}+\mathrm{Mn})$ and lowest (2366 $\mathrm{kg} / \mathrm{ha})$ in control $\left(\mathrm{T}_{0}\right)$ where micronutrient was not applied (Table 7); however, earliness index was minimum (65.50) in $\mathrm{T}_{6}$ treatment $(\mathrm{B}+\mathrm{Zn}+\mathrm{Cu}+\mathrm{Mn})$ and maximum (66.74) in control treatment (Table 3). Furthermore, mean maturity date was maximum (160.25) in $\mathrm{T}_{6}$ treatment $(\mathrm{B}+\mathrm{Zn}+\mathrm{Cu}+\mathrm{Mn})$ and minimum (159.99) in $\mathrm{T}_{0}$ (control). Treatment $\mathrm{T}_{8}$ had mean maturity date of $160.22, \mathrm{~T}_{7}$ of $160.18, \mathrm{~T}_{5}$ of 160.18 and in $\mathrm{T}_{4}$ was 160.08 (Table 7). Production rate index calculated in treatment $\mathrm{T}_{7}$ was 32.92 while in $\mathrm{T}_{6} ; \mathrm{T}_{4}$ treatment was 31.70 and 30.61 respectively. Analyzed data (Table 7) indicates that production rate index was maximum (33.64) in $\mathrm{T}_{8}$ treatment $(\mathrm{Fe}+\mathrm{B}+$ $\mathrm{Mo}+\mathrm{Zn}+\mathrm{Cu}+\mathrm{Mn}$ ) which was statistically at par with $\mathrm{T}_{7}$ treatment and minimum (27.48) in $\mathrm{T}_{0}$ (control) where micronutrient was not applied.
AJMAL, F. et al.

\section{Linear regression and correlation analysis}

Regression analysis suggests the significant dependence of seed cotton yield on plant height $\left(\mathrm{R}^{2}=\right.$ 0.87; $\mathrm{p} \leq 0.01)$. Correlation analysis also indicated a positive and linear relationship between plant height and seed cotton yield. Significant positive correlation was noted between number of leaves per plant and seed cotton yield $(\mathrm{r}=0.94 ; \mathrm{p} \leq 0.01)$ and regression analysis suggests the significant dependence of seed cotton yield on number of leaves per plant. Regression analysis also depicts the significant dependence of seed cotton yield on number of bolls per plant $\left(\mathrm{R}^{2}=\right.$ $0.82 ; \mathrm{p} \leq 0.01$ ) (Table 8). 
Effect of exogenous application...

AJMAL, F. et al.

Table 1. Effect of different micronutrients on plant height $(\mathrm{cm})$ in cotton

\begin{tabular}{|c|c|c|c|c|c|c|c|c|c|}
\hline Treatment & $45 \mathrm{DAS}$ & 54 DAS & 63 DAS & 72 DAS & $81 \mathrm{DAS}$ & 90 DAS & 99 DAS & 108 DAS & $117 \mathrm{DAS}$ \\
\hline $\mathrm{T}_{0}$ & 20.33 & $24.33 \mathrm{c}$ & $29.17 \mathrm{c}$ & $32.08 \mathrm{e}$ & $42.08 \mathrm{~d}$ & $48.42 \mathrm{~d}$ & $55.58 \mathrm{c}$ & $59.00 \mathrm{e}$ & $63.83 \mathrm{~d}$ \\
\hline $\mathrm{T}_{1}$ & 23.83 & $28.25 \mathrm{~b}$ & $34.75 \mathrm{~b}$ & $36.25 \mathrm{de}$ & $48.83 \mathrm{bcd}$ & $55.25 \mathrm{bcd}$ & $61.00 \mathrm{bc}$ & $65.42 \mathrm{de}$ & $69.42 \mathrm{~cd}$ \\
\hline $\mathrm{T}_{2}$ & 22.58 & $29.50 \mathrm{~b}$ & $37.08 \mathrm{~b}$ & $43.50 \mathrm{~b}$ & $51.58 \mathrm{bc}$ & $57.75 \mathrm{abc}$ & $65.17 \mathrm{abc}$ & 68.08 bced & $70.58 \mathrm{~cd}$ \\
\hline $\mathrm{T}_{3}$ & 22.78 & $29.17 \mathrm{~b}$ & $38.42 \mathrm{~b}$ & $41.67 \mathrm{bc}$ & $47.08 \mathrm{~cd}$ & $53.42 \mathrm{~cd}$ & $60.42 \mathrm{bc}$ & 67.42 cde & $74.42 \mathrm{abcd}$ \\
\hline $\mathrm{T}_{4}$ & 22.08 & $30.42 \mathrm{~b}$ & $37.17 \mathrm{~b}$ & $38.08 \mathrm{~cd}$ & $50.33 \mathrm{bc}$ & $60.58 \mathrm{abc}$ & $69.92 \mathrm{ab}$ & 73.75 abcd & $77.92 \mathrm{abc}$ \\
\hline $\mathrm{T}_{5}$ & 24.08 & $29.58 \mathrm{~b}$ & $36.58 \mathrm{~b}$ & $42.33 \mathrm{bc}$ & $52.25 \mathrm{bc}$ & $56.92 \mathrm{abcd}$ & $63.50 \mathrm{abc}$ & 68.67 abcde & $74.08 \mathrm{bcd}$ \\
\hline $\mathrm{T}_{6}$ & 25.08 & $28.58 \mathrm{~b}$ & $37.67 \mathrm{~b}$ & $41.17 \mathrm{bc}$ & $55.50 \mathrm{ab}$ & $64.08 \mathrm{ab}$ & $73.67 \mathrm{a}$ & $79.58 \mathrm{ab}$ & $83.92 \mathrm{ab}$ \\
\hline $\mathrm{T}_{7}$ & 24.08 & $28.50 \mathrm{~b}$ & $38.33 \mathrm{~b}$ & $41.67 \mathrm{bc}$ & $55.17 \mathrm{ab}$ & $63.42 \mathrm{ab}$ & $72.25 \mathrm{ab}$ & $79.08 \mathrm{abc}$ & $85.33 \mathrm{ab}$ \\
\hline $\mathrm{T}_{8}$ & 27.25 & $34.92 \mathrm{a}$ & $44.67 \mathrm{a}$ & $48.50 \mathrm{a}$ & $60.08 \mathrm{a}$ & $65.75 \mathrm{a}$ & $73.33 \mathrm{a}$ & $80.25 \mathrm{a}$ & $86.25 \mathrm{a}$ \\
\hline LSD at $5 \%$ & NS & 2.79 & 4.023 & 4.686 & 7.799 & 9.108 & 12.08 & 11.8 & 11.87 \\
\hline
\end{tabular}

Means sharing the common letter in a column do not differ significantly from each other at p 0.05, N.S = non significant; DAS: Days after sowing; $\mathrm{T}_{0}=\mathrm{Control}, \mathrm{T}_{1}=\mathrm{Fe}, \mathrm{T}_{2}=\mathrm{B}, \mathrm{T}_{3}=\mathrm{Mo}, \mathrm{T}_{4}$ $=\mathrm{CuSO}_{4}+\mathrm{ZnSO}_{4}+\mathrm{MnSO}_{4}, \mathrm{~T}_{5}=\mathrm{CuSO}_{4}+\mathrm{ZnSO}_{4}+\mathrm{MnSO}_{4}+\mathrm{Fe}, \mathrm{T}_{6}=\mathrm{CuSO}+\mathrm{ZnSO}_{4} ;+\mathrm{MnSO}_{4}+\mathrm{B}, \mathrm{T}_{7}=\mathrm{CuSO}_{4}+\mathrm{ZnSO}_{4}+\mathrm{MnSO}_{4}+\mathrm{Mo}_{8} \mathrm{~T}_{8}=\mathrm{CuSO}_{4}+\mathrm{ZnSO}_{4}+\mathrm{MnSO}_{4}+\mathrm{Fe}_{+}+\mathrm{B}_{+}+\mathrm{Mo}$

Table 2. Effect of different micronutrients on number of leaves per plant in cotton

\begin{tabular}{|c|c|c|c|c|c|c|c|c|c|}
\hline Treatment & $45 \mathrm{DAS}$ & $54 \mathrm{DAS}$ & 63 DAS & 72 DAS & $81 \mathrm{DAS}$ & 90 DAS & 99 DAS & 108 DAS & 117 DAS \\
\hline $\mathrm{T}_{0}$ & 14.50 & $20.25 \mathrm{~d}$ & 20.25 & $24.42 \mathrm{~d}$ & 27.25 & $33.58 \mathrm{c}$ & $39.33 \mathrm{e}$ & $42.83 \mathrm{bc}$ & 34.67 \\
\hline $\mathrm{T}_{1}$ & 15.17 & $22.08 \mathrm{~cd}$ & 24.75 & $27.25 \mathrm{~cd}$ & 33.67 & $38.50 \mathrm{bc}$ & $42.75 \mathrm{de}$ & $46.50 \mathrm{c}$ & 41.00 \\
\hline $\mathrm{T}_{2}$ & 15.17 & $24.08 \mathrm{bc}$ & 27.58 & $31.00 \mathrm{abc}$ & 35.92 & $40.75 \mathrm{ab}$ & 44.67 cde & $46.08 \mathrm{bc}$ & 42.50 \\
\hline $\mathrm{T}_{3}$ & 15.83 & $22.33 \mathrm{~cd}$ & 25.75 & $27.17 \mathrm{~cd}$ & 32.75 & $39.67 \mathrm{abc}$ & $46.00 \mathrm{~cd}$ & $48.08 \mathrm{abc}$ & 46.25 \\
\hline $\mathrm{T}_{4}$ & 16.08 & $26.25 \mathrm{a}$ & 26.75 & $31.92 \mathrm{ab}$ & 33.00 & $41.83 \mathrm{ab}$ & $47.42 \mathrm{abcd}$ & $50.67 \mathrm{abc}$ & 47.17 \\
\hline $\mathrm{T}_{5}$ & 15.83 & $23.17 \mathrm{c}$ & 23.92 & $29.33 \mathrm{bc}$ & 33.08 & $38.00 \mathrm{bc}$ & $46.17 \mathrm{bcd}$ & $51.00 \mathrm{a}$ & 49.42 \\
\hline $\mathrm{T}_{6}$ & 13.75 & $22.58 \mathrm{c}$ & 25.00 & $31.27 \mathrm{abc}$ & 34.75 & $43.50 \mathrm{ab}$ & $53.00 \mathrm{a}$ & $60.33 \mathrm{ab}$ & 60.50 \\
\hline $\mathrm{T}_{7}$ & 15.25 & $23.67 \mathrm{c}$ & 28.00 & $33.42 \mathrm{ab}$ & 36.00 & $44.08 \mathrm{ab}$ & $50.92 \mathrm{abc}$ & $56.50 \mathrm{a}$ & 59.00 \\
\hline $\mathrm{T}_{8}$ & 14.92 & $25.83 \mathrm{ab}$ & 25.42 & $34.50 \mathrm{a}$ & 39.50 & $45.83 \mathrm{a}$ & $52.58 \mathrm{ab}$ & $59.08 \mathrm{~d}$ & 62.17 \\
\hline LSD at $5 \%$ & NS & 2.109 & NS & 4.548 & NS & 6.201 & 6.444 & 10.12 & NS \\
\hline
\end{tabular}

Means sharing the common letter in a column do not differ significantly from each other at p 0.05, N.S = non significant; DAS: Days after sowing; $\mathrm{T}_{0}=\mathrm{Control}, \mathrm{T}_{1}=\mathrm{Fe}, \mathrm{T}_{2}=\mathrm{B}, \mathrm{T}_{3}=\mathrm{Mo}, \mathrm{T}$ $=\mathrm{CuSO}_{4}+\mathrm{ZnSO}_{4}+\mathrm{MnSO}_{4}, \mathrm{~T}_{5}=\mathrm{CuSO}_{4}+\mathrm{ZnSO}_{4}+\mathrm{MnSO}_{4}+\mathrm{Fe}, \mathrm{T}_{6}=\mathrm{CuSO}+; \mathrm{ZnSO}_{4}+\mathrm{MnSO}_{4}+\mathrm{B}, \mathrm{T}_{7}=\mathrm{CuSO}_{4}+\mathrm{ZnSO}_{4}+\mathrm{MnSO}_{4}+\mathrm{Mo}_{2}, \mathrm{~T}_{8}=\mathrm{CuSO}_{4}+\mathrm{ZnSO}_{4}+\mathrm{MnSO}_{4}+\mathrm{Fe}_{+}+\mathrm{B}+\mathrm{Mo}_{2}$ 
Table 3. Effect of different micronutrients on number of nodes per plant in cotton

\begin{tabular}{|c|c|c|c|c|c|c|c|c|c|}
\hline Treatment & $45 \mathrm{DAS}$ & 54 DAS & $63 \mathrm{DAS}$ & 72 DAS & 81 DAS & 90 DAS & 99 DAS & $108 \mathrm{DAS}$ & 117 DAS \\
\hline $\mathrm{T}_{0}$ & 6.00 & 9.25 & $10.75 \mathrm{c}$ & $11.25 \mathrm{f}$ & $12.33 \mathrm{c}$ & $13.58 \mathrm{~d}$ & 13.58 & $13.58 \mathrm{e}$ & 13.58 \\
\hline $\mathrm{T}_{1}$ & 8.75 & 9.92 & $11.17 \mathrm{c}$ & $12.17 \mathrm{de}$ & $13.50 \mathrm{~b}$ & $14.58 \mathrm{~cd}$ & 14.58 & $14.58 \mathrm{de}$ & 14.58 \\
\hline $\mathrm{T}_{2}$ & 7.67 & 10.00 & $11.42 \mathrm{abc}$ & $12.67 \mathrm{~cd}$ & $14.08 \mathrm{ab}$ & $14.75 \mathrm{bc}$ & 14.75 & $14.75 \mathrm{de}$ & 14.75 \\
\hline $\mathrm{T}_{3}$ & 8.17 & 9.50 & $11.17 \mathrm{c}$ & $11.83 \mathrm{ef}$ & $14.08 \mathrm{ab}$ & $15.67 \mathrm{a}$ & 15.67 & $15.67 \mathrm{~cd}$ & 15.67 \\
\hline $\mathrm{T}_{4}$ & 8.33 & 10.50 & $11.33 \mathrm{bc}$ & $11.92 \mathrm{def}$ & $13.42 \mathrm{~b}$ & $14.50 \mathrm{c}$ & 14.50 & $14.50 \mathrm{~cd}$ & 14.50 \\
\hline $\mathrm{T}_{5}$ & 7.08 & 10.17 & $12.17 \mathrm{ab}$ & $13.83 \mathrm{a}$ & $14.00 \mathrm{ab}$ & $14.50 \mathrm{c}$ & 14.50 & $14.50 \mathrm{~cd}$ & 14.50 \\
\hline $\mathrm{T}_{6}$ & 7.92 & 10.00 & $11.25 \mathrm{c}$ & $13.58 \mathrm{ab}$ & $14.08 \mathrm{ab}$ & $15.08 \mathrm{abc}$ & 15.08 & $15.08 \mathrm{bc}$ & 15.08 \\
\hline $\mathrm{T}_{7}$ & 8.58 & 11.00 & $12.25 \mathrm{a}$ & $13.25 \mathrm{abc}$ & $14.42 \mathrm{a}$ & $15.50 \mathrm{ab}$ & 15.50 & $15.50 \mathrm{ab}$ & 15.50 \\
\hline $\mathrm{T}_{8}$ & 7.92 & 10.08 & $11.50 \mathrm{abc}$ & $13.17 \mathrm{bc}$ & $14.50 \mathrm{a}$ & $15.75 \mathrm{a}$ & 15.75 & $15.75 \mathrm{a}$ & 15.75 \\
\hline LSD at $5 \%$ & NS & NS & 0.85 & 0.78 & 0.70 & 0.80 & NS & 1.16 & NS \\
\hline
\end{tabular}

Means sharing the common letter in a column do not differ significantly from each other at p 0.05, N.S = non significant; DAS: Days after sowing; $\mathrm{T}_{0}=\mathrm{Control}, \mathrm{T}_{1}=\mathrm{Fe}, \mathrm{T}_{2}=\mathrm{B}, \mathrm{T}_{3}=\mathrm{Mo}, \mathrm{T}$ $=\mathrm{CuSO}_{4}+\mathrm{ZnSO}_{4}+\mathrm{MnSO}_{4}, \mathrm{~T}_{5}=\mathrm{CuSO}_{4}+\mathrm{ZnSO}_{4}+\mathrm{MnSO}_{4}+\mathrm{Fe}, \mathrm{T}_{6}=\mathrm{CuSO}_{4}+\mathrm{ZnSO}_{4}+; \mathrm{MnSO}_{4}+\mathrm{B}, \mathrm{T}_{7}=\mathrm{CuSO}_{4}+\mathrm{ZnSO}_{4}+\mathrm{MnSO} \mathrm{Mn}_{4}+\mathrm{Mo}_{8}=\mathrm{CuSO}_{4}+\mathrm{ZnSO}_{4}+\mathrm{MnSO}_{4}+\mathrm{Fe}+\mathrm{B}+\mathrm{Mo}$

Table 4. Effect of different micronutrients on leaf area $\left(\mathrm{cm}^{2}\right)$ in cotton

\begin{tabular}{cccccccccc} 
Treatment & $45 \mathrm{DAS}$ & $54 \mathrm{DAS}$ & $63 \mathrm{DAS}$ & $72 \mathrm{DAS}$ & $81 \mathrm{DAS}$ & $90 \mathrm{DAS}$ & $99 \mathrm{DAS}$ & $108 \mathrm{DAS}$ & $117 \mathrm{DAS}$ \\
\hline $\mathrm{T}_{0}$ & 17.29 & 21.18 & $22.87 \mathrm{abc}$ & $30.03 \mathrm{~d}$ & $33.88 \mathrm{c}$ & $40.33 \mathrm{c}$ & 44.25 & $39.13 \mathrm{~d}$ & $33.80 \mathrm{~d}$ \\
$\mathrm{~T}_{1}$ & 21.67 & 23.59 & $27.84 \mathrm{ab}$ & $33.39 \mathrm{c}$ & $37.64 \mathrm{bc}$ & $43.24 \mathrm{bc}$ & 47.12 & $42.77 \mathrm{bcd}$ & $37.29 \mathrm{~cd}$ \\
$\mathrm{~T}_{2}$ & 21.12 & 24.60 & $31.65 \mathrm{bc}$ & $36.76 \mathrm{~b}$ & $44.27 \mathrm{a}$ & $48.02 \mathrm{ab}$ & 52.47 & $45.83 \mathrm{abc}$ & $38.18 \mathrm{c}$ \\
$\mathrm{T}_{3}$ & 19.98 & 26.81 & $29.80 \mathrm{a}$ & $37.11 \mathrm{~b}$ & $42.08 \mathrm{ab}$ & $47.26 \mathrm{ab}$ & 51.54 & $45.76 \mathrm{abc}$ & $38.59 \mathrm{bc}$ \\
$\mathrm{T}_{4}$ & 20.16 & 26.36 & $30.78 \mathrm{c}$ & $36.09 \mathrm{bc}$ & $44.01 \mathrm{a}$ & $51.49 \mathrm{a}$ & 51.47 & $42.11 \mathrm{~cd}$ & $38.76 \mathrm{bc}$ \\
$\mathrm{T}_{5}$ & 24.15 & 27.73 & $29.94 \mathrm{bc}$ & $38.19 \mathrm{ab}$ & $43.39 \mathrm{a}$ & $52.19 \mathrm{a}$ & 52.18 & $49.41 \mathrm{a}$ & $40.57 \mathrm{bc}$ \\
$\mathrm{T}_{6}$ & 20.57 & 26.18 & $32.30 \mathrm{a}$ & $40.52 \mathrm{a}$ & $44.03 \mathrm{a}$ & $52.68 \mathrm{a}$ & 52.89 & $48.32 \mathrm{ab}$ & $39.76 \mathrm{bc}$ \\
$\mathrm{T}_{7}$ & 20.97 & 24.92 & $26.95 \mathrm{bc}$ & $35.69 \mathrm{bc}$ & $45.63 \mathrm{a}$ & $51.85 \mathrm{a}$ & 52.94 & $47.78 \mathrm{abc}$ & $42.00 \mathrm{~b}$ \\
$\mathrm{~T}_{8}$ & 23.98 & 29.91 & $34.77 \mathrm{ab}$ & $40.91 \mathrm{a}$ & $46.33 \mathrm{a}$ & $53.49 \mathrm{a}$ & 55.55 & $50.80 \mathrm{a}$ & $45.98 \mathrm{a}$ \\
LSD at 5\% & $N S$ & NS & 5.96 & 3.25 & 4.48 & 6.69 & NS & 5.88 & 3.81
\end{tabular}

Means sharing the common letter in a column do not differ significantly from each other at p 0.05, N.S = non significant; DAS: Days after sowing; $\mathrm{T}_{0}=$ Control, $\mathrm{T}_{1}=\mathrm{Fe}, \mathrm{T}_{2}=\mathrm{B}, \mathrm{T}_{3}=\mathrm{Mo}, \mathrm{T}$ $=\mathrm{CuSO}_{4}+\mathrm{ZnSO}_{4}+\mathrm{MnSO}_{4}, \mathrm{~T}_{5}=\mathrm{CuSO}_{4}+\mathrm{ZnSO}_{4}+\mathrm{MnSO}_{4}+\mathrm{Fe}, \mathrm{T}_{6}=\mathrm{CuSO}_{4} ; \mathrm{ZnSO}_{4}+\mathrm{MnSO}_{4}+\mathrm{B}, \mathrm{T}=\mathrm{CuSO}_{4}+\mathrm{ZnSO} \mathrm{O}_{4}+\mathrm{MnSO}_{4}+\mathrm{Mo}_{8}=\mathrm{T}_{8}=\mathrm{CuSO}_{4}+\mathrm{ZnSO}_{4}+\mathrm{MnSO}_{4}+\mathrm{Fe}_{+}+\mathrm{B}+\mathrm{Mo}$ 
Table 5. Effect of different micronutrients on chlorophyll content (SPAD-502 value) in cotton

\begin{tabular}{|c|c|c|c|c|c|c|c|c|c|}
\hline Treatment & $45 \mathrm{DAS}$ & 54 DAS & 63 DAS & 72 DAS & 81 DAS & $90 \mathrm{DAS}$ & 99 DAS & 108 DAS & 117 DAS \\
\hline $\mathrm{T}_{0}$ & 37.96 & $39.25 \mathrm{c}$ & $41.29 \mathrm{f}$ & $44.06 \mathrm{e}$ & $48.14 \mathrm{abc}$ & $45.42 \mathrm{e}$ & $42.02 \mathrm{bcd}$ & $40.02 \mathrm{c}$ & $37.81 \mathrm{bc}$ \\
\hline $\mathrm{T}_{1}$ & 36.56 & $40.75 \mathrm{c}$ & $42.86 \mathrm{def}$ & $44.58 \mathrm{de}$ & $48.80 \mathrm{ab}$ & $48.78 \mathrm{~cd}$ & $44.63 \mathrm{bcd}$ & $43.52 b$ & $39.83 \mathrm{bc}$ \\
\hline $\mathrm{T}_{2}$ & 37.58 & $40.74 \mathrm{c}$ & 42.69 ef & $45.67 \mathrm{~cd}$ & $49.22 \mathrm{a}$ & $48.79 \mathrm{~cd}$ & $45.26 \mathrm{ab}$ & $44.14 \mathrm{ab}$ & $39.77 \mathrm{bc}$ \\
\hline $\mathrm{T}_{3}$ & 40.99 & $42.70 \mathrm{~b}$ & 43.58 cde & $46.01 \mathrm{c}$ & $50.86 \mathrm{c}$ & $48.00 \mathrm{~d}$ & $45.59 \mathrm{a}$ & $43.87 \mathrm{ab}$ & $40.73 \mathrm{ab}$ \\
\hline $\mathrm{T}_{4}$ & 39.47 & $43.16 \mathrm{ab}$ & 44.34 bcde & $46.55 \mathrm{c}$ & $51.80 \mathrm{bc}$ & $49.50 \mathrm{~cd}$ & $46.76 \mathrm{~d}$ & $42.94 \mathrm{bc}$ & $40.81 \mathrm{ab}$ \\
\hline $\mathrm{T}_{5}$ & 40.40 & $44.03 \mathrm{ab}$ & $44.40 \mathrm{bcd}$ & $46.22 \mathrm{c}$ & $52.35 \mathrm{c}$ & $49.96 \mathrm{~cd}$ & $45.98 \mathrm{bcd}$ & $43.41 \mathrm{~b}$ & $41.10 \mathrm{a}$ \\
\hline $\mathrm{T}_{6}$ & 38.28 & $42.53 \mathrm{~b}$ & $45.46 \mathrm{ab}$ & $49.01 \mathrm{~b}$ & $53.09 \mathrm{bc}$ & $50.76 \mathrm{bc}$ & $47.03 \mathrm{~cd}$ & $43.65 \mathrm{~b}$ & $42.63 \mathrm{c}$ \\
\hline $\mathrm{T}_{8}$ & 40.90 & $44.63 \mathrm{a}$ & $46.11 \mathrm{a}$ & $51.98 \mathrm{a}$ & $56.77 \mathrm{abc}$ & $53.14 \mathrm{a}$ & $51.47 \mathrm{abc}$ & $47.23 \mathrm{a}$ & $44.22 \mathrm{bc}$ \\
\hline LSD at $5 \%$ & NS & 1.59 & 1.70 & 1.41 & 4.13 & 2.13 & 3.95 & 3.37 & 2.73 \\
\hline
\end{tabular}

Table 6. Effect of different micronutrients number of bolls per plant in cotton

\begin{tabular}{|c|c|c|c|c|c|c|}
\hline Treatment & 72 DAS & 81 DAS & 90 DAS & 99 DAS & 108 DAS & 117 DAS \\
\hline $\mathrm{T}$ & $0.75 \mathrm{~d}$ & $0.73 \mathrm{~d}$ & $1.67 \mathrm{c}$ & $1.67 \mathrm{c}$ & $3.58 \mathrm{~d}$ & 5.17 \\
\hline $\mathrm{T}$ & $1.25 \mathrm{abc}$ & $0.89 \mathrm{~cd}$ & $3.00 \mathrm{~b}$ & $3.00 \mathrm{bc}$ & $4.50 \mathrm{~cd}$ & 6.08 \\
\hline $\mathrm{T}$ & $1.33 \mathrm{ab}$ & $1.92 \mathrm{a}$ & $3.17 \mathrm{~b}$ & $3.17 \mathrm{bc}$ & $4.92 \mathrm{~cd}$ & 6.00 \\
\hline $\mathrm{T}$ & $1.58 \mathrm{a}$ & $2.00 \mathrm{a}$ & $3.58 \mathrm{~b}$ & $3.58 \mathrm{~b}$ & $5.83 \mathrm{bc}$ & 7.25 \\
\hline $\mathrm{T}$ & $0.92 \mathrm{bcd}$ & $1.17 \mathrm{bc}$ & $3.33 \mathrm{~b}$ & $3.33 \mathrm{~b}$ & $5.50 \mathrm{bc}$ & 7.42 \\
\hline $\mathrm{T}$ & $1.08 \mathrm{bcd}$ & $1.33 \mathrm{~b}$ & $3.33 \mathrm{~b}$ & $3.33 \mathrm{a}$ & $7.33 \mathrm{bc}$ & 7.50 \\
\hline $\mathrm{T}$ & $1.00 \mathrm{bcd}$ & $1.25 \mathrm{~b}$ & $3.42 \mathrm{~b}$ & $3.42 \mathrm{a}$ & $7.67 \mathrm{ab}$ & 8.75 \\
\hline $\mathrm{T}$ & $0.83 \mathrm{~cd}$ & $1.33 \mathrm{~b}$ & $3.17 \mathrm{~b}$ & $3.17 \mathrm{~b}$ & $5.50 \mathrm{bc}$ & 7.42 \\
\hline LSD at $5 \%$ & $\begin{array}{c}0.67 \mathrm{~d} \\
0.45\end{array}$ & $\begin{array}{c}1.83 \mathrm{a} \\
0.30\end{array}$ & $\begin{array}{c}4.42 \mathrm{a} \\
0.60\end{array}$ & $\begin{array}{c}4.42 \mathrm{a} \\
1.43\end{array}$ & $\begin{array}{c}8.33 \mathrm{a} \\
1.85\end{array}$ & $\begin{array}{l}9.83 \\
\text { NS }\end{array}$ \\
\hline
\end{tabular}

$=\mathrm{CuSO}_{4}+\mathrm{ZnSO}_{4}+\mathrm{MnSO}_{4}, \mathrm{~T}_{5}=\mathrm{CuSO}_{4}+\mathrm{ZnSO}_{4} ;+\mathrm{MnSO}_{4}+\mathrm{Fe}, \mathrm{T}_{6}=\mathrm{CuSO}_{4}+\mathrm{ZnSO}_{4}+\mathrm{MnSO}_{4}+\mathrm{B}, \mathrm{T}_{7}=\mathrm{CuSO}_{4}+\mathrm{ZnSO}_{4}+\mathrm{MnSO}_{4}+\mathrm{Mo}_{8}, \mathrm{~T}_{8}=\mathrm{CuSO}_{4}+\mathrm{ZnSO}_{4}+\mathrm{MnSO}_{4}+\mathrm{Fe}+\mathrm{B}+\mathrm{Mo}$ 
Table 7. Effect of different micronutrients on number of cotton seeds per boll, seed cotton yield, earliness index (EI), mean maturity date (MMD), and production rate index (PRI) in cotton.

\begin{tabular}{cccccc}
\hline Treatment & $\begin{array}{c}\text { Number of } \\
\text { cotton seeds } \\
\text { per boll }\end{array}$ & $\begin{array}{c}\text { Seed cotton } \\
\text { yield }(\mathbf{K g} / \mathbf{h a})\end{array}$ & $\begin{array}{c}\text { Earliness } \\
\text { index }(\%)\end{array}$ & $\begin{array}{c}\text { Mean } \\
\text { maturity } \\
\text { date (days) }\end{array}$ & $\begin{array}{c}\text { Production } \\
\text { rate index } \\
\text { (g/day) }\end{array}$ \\
\hline $\mathrm{T}_{0}$ & $14.10 \mathrm{e}$ & $2366.21 \mathrm{e}$ & 66.74 & 159.99 & $27.48 \mathrm{e}$ \\
$\mathrm{T}_{1}$ & $16.27 \mathrm{~d}$ & $2407.48 \mathrm{e}$ & 66.26 & 160.09 & $27.94 \mathrm{e}$ \\
$\mathrm{T}_{2}$ & $17.07 \mathrm{~cd}$ & $2509.73 \mathrm{~d}$ & 66.55 & 160.03 & $29.14 \mathrm{~d}$ \\
$\mathrm{~T}_{3}$ & $17.17 \mathrm{~cd}$ & $2561.75 \mathrm{~cd}$ & 66.19 & 160.10 & $29.73 \mathrm{~cd}$ \\
$\mathrm{~T}_{4}$ & $17.40 \mathrm{bcd}$ & $2637.10 \mathrm{bc}$ & 66.27 & 160.08 & $30.61 \mathrm{bc}$ \\
$\mathrm{T}_{5}$ & $18.17 \mathrm{bc}$ & $2681.95 \mathrm{~b}$ & 65.82 & 160.18 & $31.11 \mathrm{~b}$ \\
$\mathrm{~T}_{6}$ & $18.43 \mathrm{~b}$ & $2733.97 \mathrm{~b}$ & 65.50 & 160.25 & $31.70 \mathrm{~b}$ \\
$\mathrm{~T}_{7}$ & $21.53 \mathrm{a}$ & $2838.02 \mathrm{a}$ & 65.80 & 160.18 & $32.92 \mathrm{a}$ \\
$\mathrm{T}_{8}$ & $21.57 \mathrm{a}$ & $2900.81 \mathrm{a}$ & 65.62 & 160.22 & $33.64 \mathrm{a}$ \\
$\mathrm{LSD}$ at $5 \%$ & 1.24 & 99.29 & $2.03(\mathrm{~N} . \mathrm{S})$ & $0.43(\mathrm{~N} . \mathrm{S})$ & 1.13 \\
\hline
\end{tabular}

Means sharing the common letter in a column do not differ significantly from each other at p 0.05, N.S = non significant; $\mathrm{T}_{0}=$ control, $\mathrm{T}_{1}=\mathrm{Fe}, \mathrm{T}_{2}=\mathrm{B}, \mathrm{T}_{3}=\mathrm{Mo}, \mathrm{T}_{4}=\mathrm{CuSO}_{4}+\mathrm{ZnSO}_{4}+\mathrm{MnSO}_{4}, \mathrm{~T}_{5}=\mathrm{CuSO}_{4}+\mathrm{ZnSO}_{4}+\mathrm{MnSO}_{4}+\mathrm{Fe}, \mathrm{T}_{6}=\mathrm{CuSO}_{4}+\mathrm{ZnSO}_{4}+\mathrm{MnSO}_{4}+\mathrm{B}, \mathrm{T}_{7}=$ $\mathrm{CuSO}_{4}+\mathrm{ZnSO}_{4}+\mathrm{MnSO}_{4}+\mathrm{Mo}, \mathrm{T}_{8}=\mathrm{CuSO}_{4}+\mathrm{ZnSO}_{4}+\mathrm{MnSO}_{4}+\mathrm{Fe}+\mathrm{B}+\mathrm{Mo}$.

Table 8. Linear regression $\left(\mathrm{R}^{2}\right)$ and correlation coefficient (r) between seed cotton yield (SCY) versus selected growth and yield components of cotton.

\begin{tabular}{lcc}
\hline Characters & Coefficient of determination $\left(\mathrm{R}^{2}\right)$ & Linear correlation coefficient $(\mathrm{r})$ \\
\hline SCY vs. plant height & $0.87^{*}$ & $0.94^{* *}$ \\
SCY vs. number of leaves per plant & $0.88^{*}$ & $0.94^{* *}$ \\
SCY vs. chlorophyll content & $0.96^{* *}$ & $0.98^{* *}$ \\
SCY vs. number of bolls per plant & $0.82^{*}$ & $0.91^{* *}$ \\
SCY vs. number of cotton seeds per boll & $0.90^{* *}$ & $0.95^{* *}$ \\
\hline
\end{tabular}

$*$ and; ** significant at 0.05 and 0.01 levels, respectively. vs $=$ versus.

\section{DISCUSSION}

The results of present investigation indicate that plant height significantly increased in treatment compared to control (Table 1). Dordas (2009) in his experiment found that plant height was increased by an average of 9 to $10 \%$ compared with the control after the application of micronutrients in cotton crop. In another study, Tahir et al. (2009) also recorded that application of chelated zinc improved the plant height significantly. Foliar application of zinc, copper, manganese, iron, boron and molybdenum have key role in increasing the number of leaves per plant (PADMA et al., 1989).

It has been observed that increase in the number of node plant might be due to the application of manganese, which plays a beneficial role in this regard (DORDAS, 2009). Application of zinc and boron significantly increased leaf area in treatment $\mathrm{T}_{8}$ compared to control (Table 4). These results are similar with the finding of Tahir et al. (2009) who found that maximum leaf area was noted in $\mathrm{ZnSO}_{4}$-DTPA followed by $\mathrm{ZnSO}_{4}$ Fulvate, and $\mathrm{ZnSO}_{4}$-EDTA, while minimum was recorded in control, where no treatment was applied. In the present study, highest leaf area $(50.8 \mathrm{~cm})$ produced in $\mathrm{T}_{8}$ treatment may be due to the application of Zinc sulphate (Table 4).

Dordas (2009) noted that chlorophyll content was increased by application of $\mathrm{Mn}$ and deficiency of $\mathrm{Mn}$ cause decline in chlorophyll content that may lead to problems of boll abscission (SILVERTOOTH et al., 1999). Application of Mn during anthesis in cotton significantly increased the number of bolls retained in the plant (DORDAS, 2009). It is therefore observed that application of zinc, iron and boron significantly increased number of bolls per plant and consequently better yield.

It has been observed previously by Ratinavel et al. (1999) that number of bolls per plant and number of cotton seeds per boll were increased in plants given combined soil application of $\mathrm{ZnSO}_{4}$ and boron. Our results are also in conformity with those of Hallikeri et al. (2002) who found that application of $\mathrm{ZnSO}_{4}+\mathrm{FeSO}_{4}$ significantly increased the seed cotton yield. Singh et al. (2015) also found that foliar application of combined micronutrient on cotton benefits to realize the higher yield. The results of present study (Table 7) are similar to those of Yaseen et al. (2013) who indicated a significant improvement in seed cotton yield with foliar application of $\mathrm{Zn}, \mathrm{B}, \mathrm{Mn}, \mathrm{Cu}$, and 
Fe on cotton grown on calcareous soils with the recommended soil applied NPK fertilizers in Pakistan. In the present study, the increase in earliness index in treatment $T_{8}$ and $T_{7}$ (Table 7) may be due to application of $\mathrm{Zn}$ and K (ZAKARIA et al., 2008).

\section{CONCLUSION}

It is inferred that foliar application could be a viable option to break the yield barrier and it might be exploited by farmers to enhance cotton productivity. The application of $\mathrm{CuSo}_{4}+\mathrm{ZnSo}_{4}+$ $\mathrm{MnSo}_{4}+\mathrm{Fe}+\mathrm{B}$ fertilization increases the seed cotton yield and yield contributing parameters particularly iron and molybdenum improved yield by increasing the number of bolls per plant.

RESUMO: Os micronutrientes desempenham um papel vital no crescimento e produtividade da cultura do algodão. Um estudo foi realizado para acessar a aplicação exógena de micronutrientes no crescimento e produção de cultura de algodão. $\mathrm{O}$ experimento foi composto de nove tratamentos como $\mathrm{T}_{0}$ (controle), $\mathrm{T}_{1}$ (Fe quelatado), $\mathrm{T}_{2}$ (B), $\mathrm{T}_{3}$ (Mo), $\mathrm{T}_{4}\left(\mathrm{CuSo}_{4}+\mathrm{ZnSo}_{4}+\mathrm{MnSo}_{4}\right), \mathrm{T}_{5}\left(\mathrm{CuSo}_{4}+\mathrm{ZnSo}_{4}+\mathrm{MnSo}_{4}+\mathrm{Fe}\right.$ quelatado $) \mathrm{T}_{6}\left(\mathrm{CuSo}_{4}+\mathrm{ZnSo}_{4}+\mathrm{MnSo}_{4}+\mathrm{B}\right), \mathrm{T}_{7}$ $\left(\mathrm{CuSo}_{4}+\mathrm{ZnSo}_{4}+\mathrm{MnSo}_{4}+\mathrm{Mo}\right)$ e $\mathrm{T}_{8}\left(\mathrm{CuSo}_{4}+\mathrm{ZnSo}_{4}+\mathrm{MnSo}_{4}+\mathrm{Fe}\right.$ quelados $\left.+\mathrm{B}\right)$. Dados sobre diferentes atributos de crescimento mostraram aumento significativo positivo com a aplicação de micronutrientes. A área foliar foi aumentada após aplicação de micronutrientes aos 99 dias após a semeadura (DAS), observando - se, então, uma tendência decrescente. Os teores de clorofila foram aumentados em 81 DAS e depois diminuíram para a colheita final. De forma semelhante, diferentes componentes de rendimento mostraram que o rendimento de algodão de sementes aumentou significativamente com a aplicação de $\mathrm{Fe}, \mathrm{B}, \mathrm{Mo}, \mathrm{Zn}, \mathrm{Cu}$ e $\mathrm{Mn}$ em comparação com o tratamento de controlo. O índice de precocidade, a data média de maturidade eo índice de taxa de produção aumentaram significativamente após o uso combinado de pulverização foliar de $\mathrm{Zn}, \mathrm{Cu}$, Mn e Mo.

PALAVRAS-CHAVE: Crescimento. Produção. Micronutrientes. Algodão.

\section{REFERENCES}

ABRO, G. H.; SYED, T. S.; UNAR, M. A.; ZHANG, M. S. Effect of application of a plant growth regulator and micronutrients on insect pest infestation and yield components of cotton. Journal of Entomology, v. 1, p. 12-16, 2004. https://doi.org/10.3923/je.2004.12.16

ALI, A.; MAHMOOD, I. A.; HUSSAIN, F.; SALIM, M. Response of rice to soil and foliar application of $\mathrm{K}_{2} \mathrm{SO}_{4}$ fertilizer. Sarhad Journal of Agriculture, v. 23, p. 15-19, 2007.

ALI, A. Effect of iron nutrient care sprayed on foliage at different physiological growth stages on yield and quality of some durum wheat (Triticum durum L.) varieties in sandy soil. Asian Journal of Crop Science, v. 4, p. 139-149, 2012. https://doi.org/10.3923/ajcs.2012.139.149

ANONYMOUS. MSTATC. Microcomputer Statistical Programme. Michigan State University, Michigan, Lansing, USA, 1986.

BAHRANI, A.; POURREZA, J. Effects of micronutrients on seed yield and oil content of Brassica napus L. cv. Talayeh. Bangladesh Journal of Botany, v. 43, n. 2, p. 231-233, 2014.

https://doi.org/10.3329/bjb.v43i2.21679

CHRISTIDIS, R. G.; HARRISON, G. J. Cotton Growing Problems. McGraw-Hill Book Company, New York, p: 16, 1955.

DONG, J. F. The yield increasing ability of spraying cotton with boron. Journal of Henan Agriculture

Science, v. 3, n. 6, 1995. 
DORDAS, C. Foliar application of manganese increases seed yield and improves seed quality of cotton grown on calcareous soil. Journal of Plant Nutrition, v. 32, p. 160-176, 2009.

https://doi.org/10.1080/01904160802609013

ECONOMIC SURVEY OF PAKISTAN. Finance Division, Economic Advisiry Wings Islamabad, p. 16-17, 2009-10.

HALLIKERI, S. S.; HALEMANI, H. L.; KHADI, B. M. Integrated foliar nutrition for yield maximization of rainfed cotton. Karnataka Journal of Agriculture Science, v. 15, p. 562-565, 2002.

LIEW, Y. A.; OMAR, S. R.; HUSNI, M. H. A.; ZAINAL, A. M. A.; ASHIKIN, N. P. A. Effects of foliar applied copper and boron on fungal diseases and rice yield. Pertanika Journal of Tropical Agriculture Science, v. 35, p .339-349, 2012.

MANGAL, P.; PRASAD, R. Nutrient management in cotton. Indian Journal of Agronomy, v. 43, p. 162-164, 1998.

MARSCHNER, H. Mineral Nutrition of Higher Plants. 2nd ed. Academic Press, London, England, 1995.

PADMA, M.; REDDY, S. A.; BABU, R. Effect of foliar sprays of molybdenum (Mo) and boron (B) on vegetative growth and dry matter production of French bean (phaseolus vulgaris L.). Journal of Research Andhra Pradesh Agriculture University, v. 17, p. 87-89, 1989.

RAB, A.; HAQ, I. Foliar application of calcium chloride and borax influences plant growth, yield, and quality of tomato (Lycopersicon esculentum Mill.) fruit. Turkish Journal of Agriculture and Forestry, v. 36, p. 695701, 2012.

RATINAVEL, K. C.; DHARMALINGAM, PANEERSEWAM, S. Effect of micronutrients on the productivity and quality of cotton seed cv. TCB 20 (Gossypium barbadense L.). Madras Journal of Agriculture, v. 86, p. 313-316, 1999.

SAJID, A.; KHAN, A. R.; MAIRAJ, G.; FIDA, M.; BIBI, S. Assessment of different crop nutrient management practices for yield improvement. Australian Journal of Crop Science, v. 2, p. 150-157, 2008.

SALEEM, M. F.; SHAKEEL, A.; BILAL, M. F.; SHAHID, M. Q.; ANJUM, S. A. Effect of different phosphorus levels on earliness and yield of cotton cultivars. Soil and Environment, v. 29, p. 128-135, 2010.

SILVERTOOTH, J. C.; EDMISTEN, K. L.; McCARTY, W. H.; SMITH, C. W.; COTHREN, J. T. Production Practices, Cotton Origin, History, Technology and Production, John Wiley Sons, New York, p. 451-488, 1999.

SINGH, S. S. Soil Fertility and Nutrient Management. Kalyani publishers, New Delhi, India: p. 38, 2003.

SINGH, K.; RATHORE, P.; GUMBER, R. K. Effects of foliar application of nutrients on growth and yield of BT cotton (Gossypium hirsutum L.). Bangladesh Journal of Botany, v. 44, n. 1, p. 9-14, 2015. https://doi.org/10.3329/bjb.v44i1.22717

STEEL, R. G. D.; TORRIE, J. H.; DICKEY, D. A. Principles and Procedures of Statistics. A biometrical approach. McGraw Hill Book Co., New York, USA. p. 400-428, 1997.

TAHIR, M.; FIAZ, N.; NADEEM, M.A.; KHALID, F.; ALI, M. Effect of different chelated Zinc sources on growth and yield of maize. Soil and Environment, v. 28, p. 179-183, 2009.

YASEEN, M.; AHMED, W.; SHAHBAZ, M. Role of foliar feeding of micronutrients in yield maximization of cotton in Punjab. Turkish Journal of Agriculture and Forestry, v. 37, p. 420-426, 2013. https://doi.org/10.3906/tar-1206-56. 
ZAKARIA, M. S.; MAHMOUD, M. H.; EL-GUIBALI, A. H.; Influence of potassium fertilization and foliar application of zinc and phosphorus on growth, yield components, yield and fiber properties of Egyptian cotton (Gossypium barbadense L.). Journal of Plant Ecology, v. 1, p. 259-270, 2008.

https://doi.org/10.1093/jpe/rtn021 